Diet, cholesterol and coronary heart disease: the Lipid Research Clinics Program

\author{
By Basil M. Rifkind, Lipid Metabolism-Atherogenesis Branch, National Heart, Lung \\ and Blood Institute, National Institutes of Health, Bethesda, Maryland, USA
}

A starting point for incriminating cholesterol in the pathogenesis of atherosclerotic coronary heart disease (CHD) is its prominent presence in the atherosclerotic plaque. Located within and without arterial wall cells it mainly takes the form of water-insoluble cholesterol esters. Cholesterol-rich lesions, similar to those of man, may be produced in an impressive variety of animal species including non-human primates, dogs, rabbits, swine and pigeons. In most instances, hypercholesterolaemia, spontaneous or induced by diet and other manipulations, underlies the development of these lesions. Recent studies on the rabbit have been particularly illuminating. The Watanabe Heritable Hyperlipidaemic (WHHL) rabbit develops severe hypercholesterolaemia due to its production of a mutant receptor for low-density lipoprotein (LDL) in a manner analogous to the human homozygote for familial hypercholesterolaemia (FHC). Atherosclerotic lesions can also be produced in rabbits by feeding them on diets high in fat which induce hypercholesterolaemia. Detailed study of rabbit atherosclerotic lesions resulting either from the heredity or diet-induced hypercholesterolaemia suggests that they are similar in major respects (Ross, 1986).

An encouraging feature of animal studies, especially of the non-human primate, are indications that atherosclerotic lesions are not irreversible. Thus regression of induced lesions has been reported in studies when lower plasma cholesterol levels have been restored, either through switching to a low-fat diet or by treatment with biliary sequestrant drugs such as cholestyramine resin (Malinow, 1981).

That cholesterol should be damaging to tissues such as the arteries comes as no surprise when its physical properties are considered. Brown \& Goldstein (1986), in their recent Nobel Laureate lecture, remind us that cholesterol is absolutely insoluble in water, a property that makes it essential in cell membranes where it modulates fluidity and maintains the barrier between cell and environment. However, its aqueous insolubility also means that when cholesterol accumulates in tissues such as the artery, it cannot be readily disposed of and its presence eventually leads to the development of an atherosclerotic plaque with potentially dangerous consequences. Brown \& Goldstein (1986) also draw attention to the potential for errant cholesterol deposition to be aggravated by the dangerous tendency of cholesterol to passively exchange between the plasma lipoproteins and cell membranes. Plasma lipoproteins such as LDL guard against this by packaging cholesterol esters in an oily core shielding them from the aqueous plasma. Levels of cholesterol must also be kept at a sufficiently low concentration to minimize passive exchange.

Approaches as diverse as tissue-culture studies, studies of LDL levels of human cord blood or of humans on low-fat diets, and prospective epidemiological studies (Brown \& Goldstein, 1986) suggest that physiological levels of plasma LDL appear to be in the range $200-600 \mathrm{mg} / \mathrm{l}$, corresponding to total plasma cholesterol levels of $1100-1500 \mathrm{mg} /$. Dramatic demonstration of the adverse effects of levels of LDL much in excess of this range is afforded by observations in human FHC in which individuals with the fortunately rare homozygous form develop severe atherosclerosis and often die from heart attack in childhood, this in the absence of other risk factors for CHD such as obesity, cigarette smoking, diabetes or hypertension. Individuals with the much more 
common heterozygous form ( 1 in 500 of the population) have lesser degrees of hypercholesterolaemia, but sufficient to result in the development of atherosclerotic CHD, albeit about 10-20 years later. Levels of total and LDL-cholesterol typical of industrial populations are considerably lower than those of the FHC heterozygote but are above physiological levels and are still sufficiently high to predispose to atherosclerotic CHD. Thus observations in over 350000 men screened for the Multiple Risk Factor Intervention Trial (MRFIT) study show a continuous graded relation between serum cholesterol levels and CHD mortality rates throughout the entire cholesterol range commencing at levels of about $1700 \mathrm{mg} / \mathrm{l}$ (Neaton et al. 1984).

\section{Clinical trials of cholesterol-lowering}

However compelling the evidence relating cholesterol to CHD is, the final link in the chain establishing the etiological significance of cholesterol is the requirement that it be directly demonstrated that cholesterol-lowering lowers the rate of CHD. Many clinical trials of cholesterol-lowering had been conducted before the Lipid Research Clinics Coronary Primary Prevention Trial (LRC-CPPT). Diet or a variety of drugs, or both, were employed, usually in individuals with clinical CHD (secondary prevention) but sometimes in healthy participants (primary prevention). Most of these studies have yielded encouraging results, but no single one could be regarded as conclusive. Such factors as the failure to randomize, absence of double-blind design, inadequate numbers and statistical problems in analysis have been held as reasons for regarding these studies as inconclusive when examined on an individual basis (Cornfield \& Mitchell, 1969). Analysis of these studies taken together has recently yielded additional information (Mann \& Marr, 1981). In an analysis of primary and secondary prevention trials using diet, in which randomization had been carried out, it was found that in general, the greater the cholesterol reduction, the greater the reduction in CHD risk; a $10 \%$ reduction in cholesterol conferred a 15 (SE 6)\% reduction in risk. In a corresponding analysis of randomized studies of drug-induced cholesterol-lowering, a $10 \%$ reduction in cholesterol was associated with a 21 (SE 5)\% reduction in CHD risk.

In considering the design of a definitive study of cholesterol-lowering, given that diet is held to play an important role in producing the higher-than-optimum cholesterol levels that lead to CHD, the ideal clinical trial would test the efficacy of diet. As mentioned previously, the results of several dietary studies, taken alone or in aggregate, strongly point to benefit - especially when the degree of cholesterol-lowering is considered-and they are consistent with the findings of drug studies. However, diet studies, which by their very nature cannot be blinded, can be criticized on the grounds that their beneficial results may have been produced by confounding; some other factor that influences CHD risk might have also changed in the groups who knew that they were taking a cholesterol-lowering diet. The National Heart, Lung and Blood Institute Task Force on Arteriosclerosis (1971) considered the possibility of a definitive dietary trial. It recommended against the conduct of a dietary study, partly because of cost, but mainly on account of the lack of feasibility of conducting a double-blind, long-term study involving the very large numbers of free-living individuals that would be required to test the diet hypothesis adequately. Decisions on the desirability of lowering plasma cholesterol through diet will continue to have to be made without direct stringent experimental proof. Those who continue to call for a trial to provide definitive proof of the effectiveness of diet are, by failing to address this issue, indefinitely postponing important decisions. 


\section{$L R C-C P P T$}

The previously mentioned considerations meant that an alternative approach was required to test whether cholesterol-lowering reduces the rate of CHD. The LRC-CPPT employed a potent, non-systemic cholesterol-lowering agent, cholestyramine, for which a placebo was available (Lipid Research Clinics Program, 1984a, $b$ ). It was a randomized, placebo-controlled, double-blind clinical trial in middle-aged men with plasma cholesterol levels in the top 5-7\% of the US cholesterol distribution. Only a few per cent of the participants were likely to have had FHC. The treatment differential resulting from the drug was about $9 \%$. A significant reduction in the primary end-point of the study, the combined incidence of definite fatal or non-fatal myocardial infarction, or both, was obtained. Corresponding reductions were seen for angina, the development of an ischaemic response to a standard exercise test and progression to coronary bypass surgery. It is important to note that the experimental comparison understates the benefit of treatment, since it includes many individuals in the cholestyramine group who, on account of poor compliance, got little or no cholesterol-lowering. When the degree of cholesterol reduction was correlated with the observed change in CHD risk a significant relation was obtained; those individuals who consistently took the full dose of the drug and obtained more than $25 \%$ reduction in cholesterol reduced their risk of CHD by almost half! Oliver (1984) has written that the LRC-CPPT findings provide 'at long last ... clear evidence that reducing very high plasma concentrations of cholesterol and low density lipoprotein-cholesterol lowers the incidence of coronary heart disease'.

The LRC-CPPT investigators also correlated the results of ten studies whose design features and results allowed comparison with the LRC-CPPT (Lipid Research Clinics Program, 1984b). Although most of these trials, considered individually, did not report a statistically significant treatment benefit with respect to the predefined primary endpoint, the calculated reductions in CHD incidence demonstrated beneficial trends in eight of the ten studies. Results of most of these studies (including the LRC-CPPT) can be fitted to a regression line relating cholesterol reduction to decreased $\mathrm{CHD}$ risk based on Cox proportional hazards analysis of CHD incidence within the LRC-CPPT cholestyramine group (in which a $10 \%$ change in total cholesterol translated into a $23 \cdot 1 \%$ reduction in $\mathrm{CHD}$ risk). Thus the major clinical trials of cholesterol-lowering, including the LRC-CPPT, broadly agree with one another in showing that the greater the degree of cholesterol reduction, the greater the reduction in risk. The impressive consistency of the CPPT results with those of the Oslo heart study, the WHO clofibrate trial and the Los Angeles Veterans Administration dietary trial was noted by Oliver (1984). These cholesterol-CHD risk relations are also strikingly close to what was predicted from the study in Framingham, USA, and other observational studies.

\section{Other clinical trials}

In addition to the LRC-CPPT the results of other studies point to the benefits of cholesterol-lowering. That dietary-induced cholesterol-lowering is beneficial in hypercholesterolaemic men was reported by the Oslo study, in which men assigned to receive a diet- and smoking-intervention programme had a $47 \%$ lower incidence of fatal and non-fatal myocardial infarction compared with a control group (Hjermann et al. 1981). Most of the benefit was attributed to the reduction in total cholesterol.

Further support for the effect of diet comes from a report from the MRFIT study (Cutler et al. 1985); although the cholesterol differential between the specialintervention and usual-care groups was only $2-3 \%$ and limited what could be learned by comparing the two groups, Cox regression analysis showed that, in the former group, the 
degree of cholesterol reduction significantly correlated with the CHD death rate.

An interesting finding has recently emerged from follow-up of the nicotinic acid treated group in the Coronary Drug Project (CDP) (Canner, 1985). It was previously reported that this group experienced a significant $25 \%$ reduction in the incidence of non-fatal myocardial infarction, unaccompanied by significant changes in fatal myocardial infarction or total mortality. However, analysis of a post-trial follow-up of CDP participants has now demonstrated a reduction in total mortality in the nicotinic acid treated subjects within 2 years after the treatment period ended. It is reasonable to expect, on pathophysiological grounds, that the benefits of a prolonged period of cholesterol-lowering would be maintained for some time even if treatment were stopped.

Given that the various studies used diet or drugs for primary or secondary prevention, and that some recruited individuals from a broad range of cholesterol levels (for example, in the CDP the cholesterol level was not an entry criterion), the trials themselves address directly some aspects of the generalizability of the benefits of cholesterol-lowering. The compatibility of the findings of these trials with those predicted from the prospective observational studies on various population groups also suggests the broad applicability of the findings. Furthermore, the quantitative relation between cholesterol-lowering and CHD risk seen in the LRC-CPPT is of particular importance given its consistency with what had been predicted from observations made in prospective population-based epidemiological studies. The results of the LRC-CPPT, those of other clinical trials of cholesterol-lowering and the findings of the prospective epidemiological studies are in good general agreement and indicate, as a rough rule-of-thumb, that each $1 \%$ reduction in plasma cholesterol results in a $2 \%$ reduction in CHD incidence.

In considering the implications of the LRC-CPPT, several points are worth bearing in mind:

(1) The cut-off point for entry into the trial was $2650 \mathrm{mg}$ cholesterol/l, corresponding to the top $5-7 \%$ of the US cholesterol distribution (and to about $20 \%$ of the UK distribution). This cut-off point was chosen to select individuals at sufficiently high risk to generate sufficient end-points for a statistically meaningful study. Since risk is continuous and graded throughout the cholesterol distribution it is reasonable to expect that lesser degrees of elevated cholesterol would also benefit from cholesterol reduction, as is indeed suggested by some of the other trials of cholesterol-lowering.

(2) As indicated previously, the average $19 \%$ reduction in CHD risk understates the potential benefit of cholesterol-lowering; those individuals who adhere to treatment and achieve substantial reductions in cholesterol levels may reduce the risk by about half.

(3) There is little reason to believe that only resin-induced cholesterol (and LDL) lowering is beneficial. Aggregate analysis of a variety of trials using different drugs or diets suggests benefit. In addition, several studies suggest that LDL reduction through diet would be effective, cheaper and likely to be safer than the use of drugs. In fact one would be hesitant to advocate drug therapy in individuals with lesser degrees of hypercholesterolaemia. Also, evidence from several clinical trials, the LRC-CPPT placebo group and the Oslo trial suggests that dietary treatment is of benefit.

\section{National Institutes of Health (NIH) Consensus Conference}

The NIH Consensus Development Conference on Lowering Blood Cholesterol to Prevent Heart Disease was asked to review all the evidence pertaining to cholesterol and CHD including, but by no means restricted to, the LRC-CPPT. The panel unanimously concluded that the evidence supporting a causal relation between plasma cholesterol levels and CHD comes from a wealth of congruent results of genetic, experimental, 
pathologic, epidemiologic and intervention studies. They concluded that the findings established beyond any reasonable doubt the close relation between elevated cholesterol levels and CHD. However, they emphasized that the plasma cholesterol level is not the only cause of CHD (NIH Consensus Panel on Lowering Blood Cholesterol to Prevent Heart Disease, 1985).

The Consensus Panel set target levels of $2000 \mathrm{mg}$ cholesterol/ for adults over age 30 years and $1800 \mathrm{mg} / \mathrm{l}$ for adults of 30 years and below. It then proceeded to recommend a dual strategy designed to markedly decrease the incidence of CHD. One arm consisted of defining individuals at so-called high and moderate risk for CHD on account of especially high cholesterol levels (Table 1); these groups involve one in four of the US population and a higher proportion of the UK population. Management of these 'at-risk' subjects requires the involvement of physicians and dietitians for their evaluation, diagnosis and management. Diet is the first approach to the treatment of each of these groups. Only in the highest-risk group should additional drug therapy be contemplated if necessary. The other arm, directed to the rest of the population and relatively sparing of medical resources, was to moderately reduce the total saturated fat and cholesterol intake of the population as a whole. This latter recommendation broadly resembles those made by over forty official bodies and experts from many countries over the past 15-20 years, in which the main recurring points are less total fat, less saturated fat and, in most cases, less dietary cholesterol and partial replacement of saturated by polyunsaturated fat (Truswell, 1983). The recent COMA report supports many of these recommendations (Department of Health and Social Security, 1984).

It should be emphasized that these two approaches are mutually compatible and should not be regarded as alternatives. Concentration on one arm to the exclusion of the other would result in the neglect of a large proportion of the ischaemic heart disease in the population attributable to higher than optimum cholesterol levels in that group.

\section{National Cholesterol Education Program}

Recognizing that the implementation of these recommendations requires improved professional and public education regarding the role of cholesterol and its control, the National Heart, Lung and Blood Institute has recently initiated the NCEP (Lenfant, 1986). The National Coordinating Committee for this programme has assembled representatives from twenty-four of the major national professional organizations in the clinical, public health and nutrition fields (Table 2). Its main aim is to further reduce the incidence of CHD mortality and morbidity through cholesterol-lowering. Taking as its model the highly successful National High Blood Pressure Education Program, the NCEP seeks to promote and coordinate the many activities that relate to attempts to improve the detection, diagnosis and treatment of high blood cholesterol. In planning the NCEP it has been recognized that many segments of industry have an important role to play, especially those in the food producing, processing and marketing sectors with whom extensive consultation has taken place.

Table 1. Plasma cholesterol values for adults at moderate and high risk of coronary heart disease

\begin{tabular}{|c|c|c|c|c|}
\hline \multirow{2}{*}{$\begin{array}{l}\text { Age } \\
\text { (years) }\end{array}$} & \multicolumn{2}{|c|}{ Moderate risk } & \multicolumn{2}{|c|}{ High risk } \\
\hline & $\mathrm{mmol} / \mathrm{l}$ & $\mathrm{mg} / \mathrm{l}$ & $\mathrm{mmol} / \mathrm{l}$ & $\mathrm{mg} / \mathrm{l}$ \\
\hline $20-29$ & $5 \cdot 17$ & 2000 & $5 \cdot 69$ & 2200 \\
\hline $30-39$ & 5.69 & 2200 & $6 \cdot 21$ & 2400 \\
\hline 40 and over & $6 \cdot 21$ & 2400 & 6.72 & 2600 \\
\hline
\end{tabular}




\section{Table 2. National Cholesterol Education Program Coordinating Committee member organizations}

American Academy of Family Physicians

American Academy of Pediatrics

American Association of Occupational

Health Nurses

American College of Cardiology

American College of Physicians

American College of Preventive Medicine

American Dietetic Association

American Heart Association

American Hospital Association

American Medical Association

American Nurses Association

American Occupational Medical Association
American Osteopathic Association

American Pharmaceutical Association

American Public Health Association

American Red Cross

Association of Black Cardiologists

Association of Life Insurance

Directors of America

Association of State Territorial Health Officials

Citizens for Public Action on Cholesterol

National Black Nurses Association

National Heart, Lung and Blood Institute

National Medical Association

Society for Nutrition Education

\section{REFERENCES}

Brown, M. S. \& Goldstein, J. L. (1986). Science 232, 34-47.

Canner, P. L. (1985). American Heart Association Council on Epidemiology, March, Abstr.

Cornfield, J. \& Mitchell, S. (1969). Archives of Environmental Health 19, 382-391.

Cutler, J. A., Neaton, J. D., Hulley, S. B., Kuller, L., Paul, O. \& Stamler, J. (1985). Preventive Medicine 14, 293-311.

Department of Health and Social Security (1984). Diet and Cardiovascular Disease. Committee on Medical Aspects of Food Policy. Report of the Panel on Diet in Relation to Cardiovascular Disease. London: H.M. Stationery Office.

Hjermann, I., Velve Byre, K., Holme, I. \& Leren, P. (1981). Lancet ii, 1303-1310.

Lenfant, C. (1986). Circulation 73, 855-856.

Lipid Research Clinics Program (1984a). Journal of the American Medical Association 251, 351-364.

Lipid Research Clinics Program (1984b). Journal of the American Medical Association 251, 365-374.

Malinow, M. R. (1981). Circulation 64, 1-3.

Mann, J. I. \& Marr, J. W. (1981). Lipoproteins, Atherosclerosis, and Coronary Heart Disease, pp. 197-210 [N. E. Miller and B. Lewis, editors]. Amsterdam: Elsevier/North Holland Biomedical Press.

National Heart, Lung and Blood Institute Task Force on Arteriosclerosis (1971). Arteriosclerosis, vol. 1, pp. 72-137. Washington DC: Department of Health, Education and Welfare Publication (NIH).

Neaton, J. D., Kuller, L. H., Wentworth, D. \& Borhani, N. O. (1984). American Heart Journal 108, 759-770.

NIH Consensus Panel on Lowering Blood Cholesterol to Prevent Heart Disease (1985). Journal of the American Medical Association 253, 2080-2086.

Oliver, M. F. (1984). British Medical Journal 288, 423-424.

Ross, R. (1986). New England Journal of Medicine 314, 488-500.

Truswell, A. S. (1983). Food Technology in Australia 35, 498-502. 